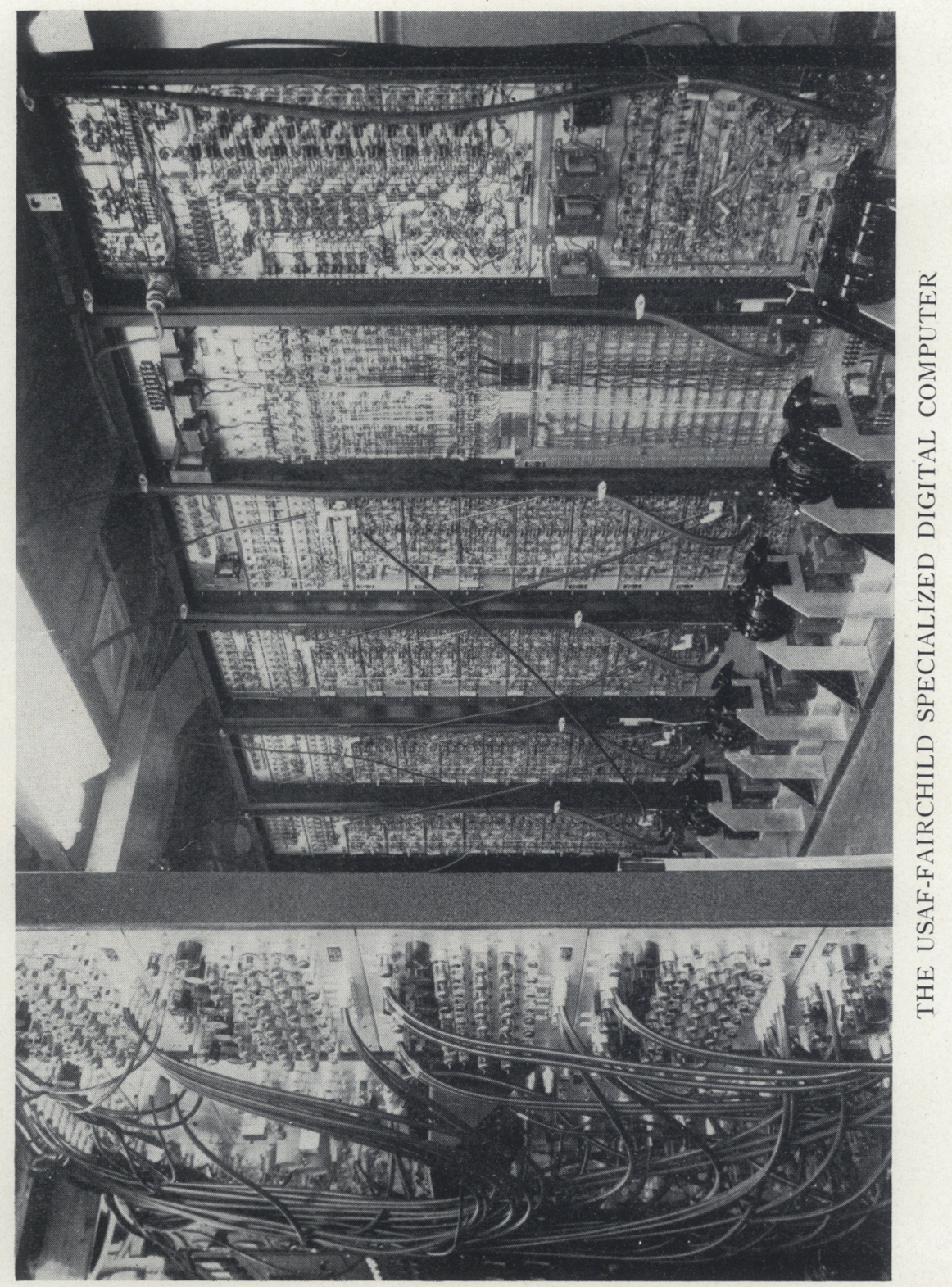




\section{Addendum to a Guide to Tables on Punched Cards}

$A$ Guide to Tables on Punched Cards was published in October, 1951 [MTAC, v. 5, p. 185-212]. In July, 1952 [MTAC, v. 6, p. 204-205], an announcement was made about a forthcoming addendum to this guide, and information was requested concerning any new contributions or necessary corrections. This addendum and list of corrections is now given below. In addition, information is supplied in Section 2 about keypunching of important tables that is now in progress at various laboratories.

It might be interesting to observe that the supply of new tables on punched cards is rather small, compared with the activity in mathematical computing that has been stimulated by the increasing number of high-speed calculators. It is possible that this foreshadows the replacement of punched cards by more satisfactory tools, which may become available even to laboratories of relatively small size. For the present, however, punched cards are still very much in use and it is deemed desirable to keep the record of them up-to-date.

\section{Additions:}

Source Available at

$[(18)] \quad(18) \mathrm{C}$

$[(18)] \quad(18) \mathrm{C}$

$2.6 \sqrt{x}: x=1(1) 9999 ; 9 \mathrm{D} ; 4000$ cards $(x=0001,1001,2001$ on same card, etc.)

[(24)] (24)C

2.9 Sums related to powers of complex numbers.

$$
\begin{aligned}
& U_{n}=8^{n} / n ! ; T_{n}=U_{n} / n \\
& R_{k}+i S_{k}=\sum_{n=1}^{\infty} U_{k+4 n}, \\
& P_{k}+i Q_{k}=\sum_{n=1}^{\infty} T_{k+4 n}, \\
& R_{k}, S_{k}, P_{k}, Q_{k}, k=1(1) 4 ; 10 \mathrm{D} \\
& z=x+i y ; x, y=0(.1) 3.1 ; \\
& y \leq x .
\end{aligned}
$$

Useful for computing the exponential functions and their integrals in the complex plane. 
Source Available at [(24)] (24)C, (73)UMT 145[D,F]

$[(67)]$

$[(67)]$

$[(67)]$

$[(67)]$

$[(67)]$

$[(67)]$

$[(23)-(18)](18) \mathrm{C}$

[(18)]

$[(16)-(4)]$

$[(25)]$

$[(5)] \quad(18) \mathrm{C}$

$[(66)-(4)]$

$[(66)-(4)]$

$[(66)-(4)]$
Description of Tables

7.1 Cyclotomic cosines $2 \cos (2 \pi k / p):$

$k=1(1)\left(\frac{p-1}{2}\right) ; 20 \mathrm{D}$

$p$ an odd prime $<100$.

517 values

(Computed under supervision of D. H. LEHMER.)

$7.4 \sin x, \cos x, \Delta, \Delta / 60$ :

$x=0\left(1^{\prime}\right) 90^{\circ} ; 8 \mathrm{D}$

(See Note 1 at end of section.)

$7.4 \sin x, \cos x, D^{1}, D^{2}$ :

$x=0^{\prime \prime}\left(1000^{\prime \prime}\right) 129600^{\prime \prime} ; 8 \mathrm{D}$

(See Note 1 at end of section.)

$7.5 \sin x, \cos x, \Delta$ :

$x=0^{\circ}\left(100^{\circ}\right) 172800^{\circ} ; 5 \mathrm{D}$

$7.5 \sin x, \cos x, \Delta$ :

$x=0\left(0^{h} .01\right) 11^{h} .99 ; 4 \mathrm{D}$

$9.10 y=\arcsin x, D^{1}, D^{2}$ :

$x=0(.01) 0.52 ; y$ to $6 \mathrm{D}$ of a degree

$9.15 y=\arctan x$ :

$x=0(.01) 1 ; y$ to $7 \mathrm{D}$ of an hour

$10.0 \quad e^{-x}: x=0(.01) 30 ; 14 \mathrm{D}$

$10.0 \quad e^{-x}: x=0(.001) 2$;

also $x=0(.01) 6(.1) 14.6 ; 5 \mathrm{D}$

$14.61 \frac{1}{\Gamma(z)}=U+i V: z=x+i y$;

$x=-.5(.01) .5 ; y=0(.01) 1 ; 6 \mathrm{D}$

14.9 Incomplete Beta function

$\beta_{x}(p, q): x=1, p \leq q=0.05$

(.05) $10 ; 6 \mathrm{~S}$

$x=\frac{1}{2} ; p=q=.05(.05) 10 ; 6 \mathrm{~S}$

See Bureau of Mines R I 4917, in press, for details and tabular entries.

$17.1 J_{0}(x), J_{1}(x), \Delta, \Delta^{2}, \Delta^{8}, \Delta^{4}$ :

$x=25.01(.01) 99.9 ; 18 \mathrm{D}$

$18.1 \quad I_{0}(x), \delta^{2}: x=0(.001) 5 ; 9 \mathrm{~S}$

$18.3 \quad K_{0}(x), \delta^{2}: x=.01(.01) 5 ; 8 \mathrm{~S}$

$\left\{18.3 \quad e^{-x} I_{0}(x), \delta^{2}, e^{x} K_{0}(x), \delta^{2}:\right.$

$\{18.4 x=5(.01) 10(.1) 20 ; 8 \mathrm{~S}$ 
Source Available at

[(24)] (24)C

$[(24)-(12)](24),(12) \mathrm{C}$

$[(4)] \quad(4) \mathrm{C}$

$[(15)]$

(15)

$[(18)] \quad(18) \mathrm{C}$

$[(4)-(4)]$

$[(14)-(4)] \quad(4)$
Description of Tables

$$
\begin{aligned}
20.294 f(x) & =\int_{0}^{x} A i(-t) d t \\
F(x) & =\int_{0}^{x} f(t) d t
\end{aligned}
$$

where $\operatorname{Ai}(t)$ is the familiar Airy integral:

$x=-2(.01) 5 ; 8 \mathrm{D}$ in $f(x)$;

$7 \mathrm{D}$ and $8 \mathrm{D}$ in $F(x)$, with the eighth place uncertain. Computed by Elmer E. Osborne.

$22.56 e^{-x} L_{n}(2 x)$, where $L_{n}(2 x)$ is the Laguerre polynomial:

$$
\begin{aligned}
& n=0(1) 12, \\
& x=0(.1) .5(.5) 10(1) 20 ; 10 \mathrm{D} \\
& n=13(1) 20 ; x=0(.5) 10(1) 20 ; \\
& 10 \mathrm{D}
\end{aligned}
$$

Computed under supervision of C. Lanczos.

22.68 Fermi functions (see definitions in note 2 at end of section).

Table 1.

$\phi_{0}(p, z):|z|=2(1) 96$;

$p=0(.1) 1(.2) 3(.5) 5(1) 9(2) 15$

$|z|=2(1) 25 ; p=20,25$; to about $4 \mathrm{~S}$

Table 2.

$\frac{p}{W} \frac{F_{v}}{F_{0}}: \nu=1,2,3$; same range as Table 1, except that $p \geq 0.1$.

25.1 Random digits. Random with respect to card column, as well as with respect to digits in col. Made by Jack Sherman. See Note 4.

25.1 8-digit random numbers computed by Lehmer's rule- 9 numbers per card, 3,000 cards

25.21 Random variates from distribution $\exp (-y)$. [A number $r$ was chosen at random from 4-decimal numbers. Then $-\log r=y]$. 140,000 cards.

25.31 Random direction cosines from a spherically symmetric distribution. Three-dimensional, $l, m, n$, to 3 decimals. 70,000 . 
Source Available at

$[(25)]$

[25]

Northrop (73)UMT 138[F]

[(24)] (24)C, UMT $146[\mathrm{~F}, \mathrm{~L}]$

$[(18)] \quad(18) \mathrm{C}$
Description of Tables

26.1 Tables relating to hydrodynamics

Tables of velocity of steady laminar flow in channels of rectangular cross-section (see definition in note 3 at end of section).

$w(x, y, \sigma)$ to $6 \mathrm{D}$ for $x, y, \sigma$ ranging between 0 and 1 .

27.1 Products of powers of small primes $N=2^{a} 3^{b} 5^{\circ} 7^{d}: a=0(1) 11$;

$b=0(1) 8 ; c=0(1) 5 ; d=0(1) 4$; exact.

Computed by R. A. Johnson, Northrop Aircraft, Inc., Hawthorne, Calif.

27.7 Kloosterman sums

$$
\begin{aligned}
& s_{p}(k)=\sum_{n=1}^{p-1} \exp \{2 \pi i(k n+\bar{n}) / p\} \\
& n \bar{n} \equiv 1(\bmod p) \\
& k=1(1)(p-1), \quad p \text { a prime } \\
& <100,19 \mathrm{D} . \\
& 1034 \text { cards. Computed under } \\
& \text { supervision of D. H. LEHMER. }
\end{aligned}
$$

28.1 Astronomy. Julian date to calendar date 1639 (30 Julian days) $2060 ; 3,846$ cards

Note 1. The Admiralty Computing Service is now at an end; but the punched cards listed under [67] are available.

Definitions: $D^{2}=\frac{1}{4}\left(\delta_{0}{ }^{2}+\delta_{1}^{2}\right) ; D^{1}=\delta_{\frac{1}{1}}-D^{2}$.

(If $h$ is the interval between two consecutive arguments, then $f\left(x_{0}+p h\right)$ $=f_{p}=f_{0}+p D^{1}+p^{2} D^{2}$.)

Note 2. Fermi functions 22.68

$$
\begin{aligned}
F_{\nu}= & {\left[\frac{(2 \nu+2) !}{\nu !}\right]^{2}\left(2 p r_{0}\right)^{2\left(\iota_{\nu}-\nu-1\right)} \frac{e^{\pi \nu}\left|\Gamma\left(s_{\nu}+i y\right)\right|^{2}}{\Gamma^{2}\left(2 s_{\nu}+1\right)} ; } \\
\phi_{0}(p, z)=\frac{p}{W}\left(\frac{1+s_{0}}{2}\right) F_{0}(p, z) ; & \\
s_{\nu}= & \sqrt{(\nu+1)^{2}-\alpha^{2} z^{2}} ; \quad y=\alpha z W / p ; \quad W=\sqrt{p^{2}+1} ; \quad r_{0}=\frac{1}{2} \alpha A^{t} ; \\
& \alpha=1 / 137.03 \\
z= & \text { atomic number of } \beta \text { emitter } ; z>0 \text { for electrons; } z<0 \text { for positrons. }
\end{aligned}
$$


$A=$ mass number of $\beta$ emitter; $p=$ momentum of $\beta$ particle in $m c$ units $W=$ total energy in $m c^{2}$ units; $r_{0}=$ nuclear radius in $h / m c$ units.

Write to (4) for further details.

Note 3. Tables relating to hydrodynamics 26.2

where

$$
w(x, y, \sigma)=1-x^{2}+\frac{4}{\pi^{3}} \sum_{n=0}^{\infty}(-1)^{n+1}\left(n+\frac{1}{2}\right)^{-3} \cos \left(u_{4}\right) F(n, y, \sigma)
$$

$$
\begin{aligned}
& \qquad F(n, y, \sigma)=\left(\exp u_{1}+\exp u_{2}\right) /\left(1+\exp u_{3}\right) \\
& u_{1}=-\left(n+\frac{1}{2}\right)(1-y) \frac{\pi}{\sigma} ; u_{2}=-\left(n+\frac{1}{2}\right)(1+y) \frac{\pi}{\sigma} ; u_{3}=-(2 n+1) \frac{\pi}{\sigma} ; \\
& u_{4}=\left(n+\frac{1}{2}\right) \pi x \text {. See Bureau of Mines R.I. } 4885 \text { (July 1952) for further } \\
& \text { details. }
\end{aligned}
$$

Note 4. The random digits were prepared by W. F. Brown, JR., of Sun Physical Laboratory, Newton Square, $\mathrm{Pa}$., with the aid of random digits of the RAND Corp.

Corrections: [MTAC, v. 5, 1951]

p. 190, Source 55(b), for $\sinh F-1$ read $\sinh F-F$.

p. 196, 7.1 Items 2 and 10 under this listing, (18)C: for $x=0(.001) 1.571$; $7 \mathrm{D}$ read $x=1(.001) 1.228$.

p. 201, 10.0 Item $9,1.13$. for $14 \mathrm{~S}$ and $18 \mathrm{~S}$ read $15 \mathrm{~S}$.

p. 201, 11.6 Add the remark: "See also 28.3."

p. $203,14.6$ for $x=9(.1) 10 ; y=0(.1) 10 ; 14 \mathrm{D}$ or $15 \mathrm{D}$ read $x, y=0(.1) 10$; $12 \mathrm{D}$ and $14 \mathrm{D}$. (Extension previously in progress now completed.)

p. 205, 17.1 Item 3, add "Available at (18)."

p. 209, 25.2 Tabulation of $x^{2}$ not available at (4).

p. 209, 25.3 for Cards 0(1)14,566 read Cards 0(1)13,000.

p. 209, 25.3 for 9.4247 read 1.5707 .

p. 211, 28.2 Kepler's equation: for $E=M-e \sin E$ read $M=E-e \sin E$.

Keypunching in progress.

(a) At [24]: $I_{\nu}(x), \pm \nu=\frac{1}{3}, \frac{2}{3}, \frac{1}{4}, \frac{3}{4}$

(b) At Point Mugu, under supervision of Donald Dufford, NAMTC, $J_{\nu}(x)$, for same values of $\nu$ as those above. Thus both volumes of the NBS Tables of Bessel functions of fractional order are now being keypunched. 
(c) At [24]: $I_{n}(x)$, based on manuscript of BAAS now in print.

(d) [24]: Spherical Bessel functions. Keypunched but not checked.

(e) At NOTS, Kenneth C. Rich and Charles Ricker, China Lake Pilot Plant, China Lake, California. Bessel functions $y_{n}(x)$ and $Y_{n}(x), n \leq 20$, BAAS, v. 10 .

Readers are again requested to review their keypunching loads, for possible keypunching of other basic mathematical tables during spare hours. Any one who can undertake a part of this work is requested to communicate with the undersigned.

Gertrude Blanch

Everett C. Yowell

National Bureau of Standards

Los Angeles 24, Calif.

\section{The Sieve Problem for All-Purpose Computers}

Introduction. The term all-purpose digital computer is often used to indicate a computing machine capable of performing the rational operations using addition and multiplication as basic functions of the arithmetic unit. Even when these operations are supplemented by some discrimination and "extract" commands, for dealing directly with the digits of numbers, there are a number of processes to which such a machine is not well suited. This includes even finite processes that are combinatorial in nature. Perhaps the most well-known of these processes is the sieve process. Although special equipment has been constructed for carrying out this algorithm ${ }^{1}$ we shall not describe it here. Our purpose is to indicate how the all-purpose computer may be programmed to compete with sieve machines.

Let us first state the general problem to be solved by the sieve process. Let $m_{1}, m_{2}, \cdots, m_{k}$ be a set of $k$ positive integers which, for the purposes we have in mind, may be assumed to be relatively prime in pairs. For each $m_{i}$ we consider $n_{i}$ distinct arithmetical progressions or linear forms in the variable $x$ which we denote by

$$
P_{i j}(x)=m_{i} x+a_{i j}\left\{\begin{array}{l}
i=1,2, \cdots, k \\
j=1,2, \cdots, n_{i}
\end{array} .\right.
$$

We may assume that, for $i$ fixed, the $a_{i j}$ are distinct non-negative integers less than $m_{i}$. The problem is to find all integers $N$ between given limits, say

$$
A \leq N<B
$$

such that each $N$ belongs to $k$ arithmetical progressions.

The number $k$ is called the width of the problem, the $k$ numbers $m_{i}$ will be called the moduli of the problem and the numbers $a_{i j}\left(j=1 \cdots n_{i}\right)$ the admissible remainders for the modulus $m_{i}$. The solution $N$, or even the number of solutions, is an exceedingly complicated function of the given parameters $m_{i}, a_{i j}, A, B$. There are two extreme cases which may be mentioned. 